\title{
BROWNIAN MOTION IN A FIELD OF FORCE AND THE DIFFUSION THEORY OF CHEMICAL REACTIONS. II
}

\author{
by H. C. BRINKMAN \\ Centraal Laboratorium T.N.O., Delft, Nederland
}

\section{Synopsis}

H. A. Kra $\mathrm{mers}^{1}$ ) has studied the rate of chemical reactions in view of the Brownian forces caused by a surrounding medium in temperature equilibrium. In a previous paper ${ }^{2}$ ) the author gave a solution of Kramers' diffusion equation in phase space by systematic development. In this paper the general problem of the shuttling forward and back of a particle between two potential holes is studied.

It is found that the reaction rates depend exponentially on the ratio of activation energy to $\mathrm{kT}$. This remains true for a reaction proceeding simultaneously in both directions. The form of temperature dependence of the non-exponential factor is determined by the potential curve. Analogous results are obtained for a system containing many degrees of freedom.

1. Introduction. $\mathrm{H} . \mathrm{A} . \mathrm{Kra} \mathrm{mers}^{1}$ ) has studied the rate of chemical reactions at the following model. A particle moves in an external field of force, but - in addition to this - is subject to the irregular forces of a surrounding medium in temperature equilibrium (Brownian motion). Originally the particle is caught in a potential hole, but it may escape in the course of time by passing over a potential barrier. Assuming the validity of The Einstein theory of Brownian motion, Kramers derived a diffusion equation in phase space, describing the above process. Assuming a stationary diffusion current, Kramers was able to derive expressions for the reaction rate and the compare these expressions to those obtained from the so called transition state method.

In a previous paper ${ }^{2}$ ) the author gave a solution of Kramers' diffusion equation in phase space by systematic development. It was shown that in this development Smoluchowski's diffusion equation in coordinate space appears as a first approximation. Higher approximations were given too and their magnitude was evaluated.

In this paper a model consisting of two potential holes, separated by a potential barrier is studied and the reaction rates in both directions are calculated.

2. The mathematical method. A particle moves in a one dimensional 
potential field $U(q)$ of the general form as sketched in fig. 1. The particle is subject to an external force $F(q)$

$$
F=-\mathrm{d} U / \mathrm{d} q
$$

A chemical reaction is represented by a transition of the particle from state 1 via the transition state $t$ to the final state 2 or vice versa.

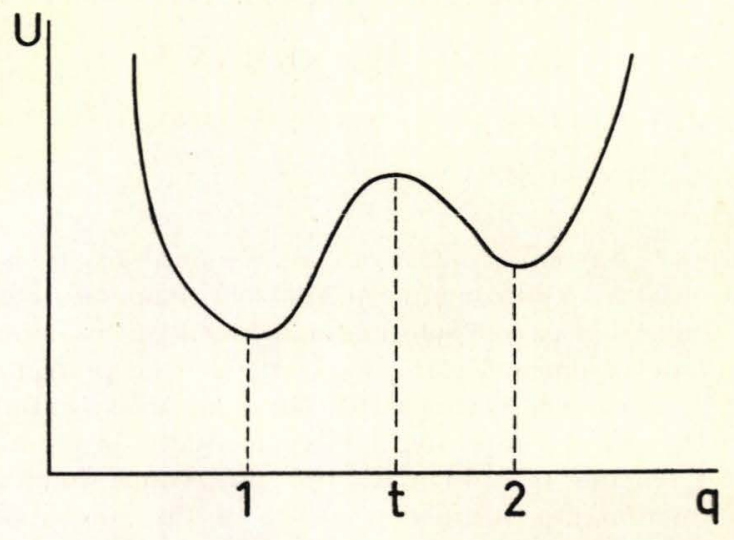

Fig. 1.

The energy necessary for the passage through the transition state $t$ is supplied by a medium of temperature $T$ and viscosity $\eta$ in which the particle is embedded. The diffusion process may then be described in a first approximation by the Smoluchowski diffusion equation ( $S$-equation) which has the form of an equation of continuity

$$
\partial \sigma / \partial t=-\partial w / \partial q
$$

where $\sigma$ is the particle density along the $q$-coordinate and $w$ is the $S$-current. This current $w$ consists of two parts, one due to diffusion and one due the systematic velocity $v$ of the particle

$$
w=-D \partial \sigma / \partial q+v \sigma
$$

For spherical particles (radius $a$ and mass $m$ ) $D$ and $v$ may be expressed as

$$
\begin{aligned}
D & =k T / 6 \pi \eta a \\
v & =F / 6 \pi \eta a
\end{aligned}
$$

It is preferred however to introduce two other quantities, viz the friction factor $f$ and the relaxation time $\tau$

$$
\begin{gathered}
f=6 \pi \eta a \\
\tau=m / 6 \pi \eta a
\end{gathered}
$$

The friction factor $f$ determines the friction between particle and medium. The meaning of the relaxation time $\tau$ may be understood by observing that 
a free particle $(F=0)$ subject to Brownian forces starting with initial velocity $\dot{q}_{0}$ travels a mean distance $\dot{q}_{0} \tau$ during an infinite time interval. In other words $\tau$ gives an estimate of the time necessary to set up a Boltzmann distribution in the gas kinetic velocities of the particles.

The $S$-current may now be written as

$$
w=f^{-1}(-k T \partial \sigma / \partial q+F \sigma)
$$

This expression may be considered as a first approximation. A second approximation was derived from Kramers' diffusion equation in phase space ${ }^{2}$ ). The correction terms read

$$
w_{2}=\tau \frac{\partial}{\partial q} f^{-2}(-k T \partial / \partial q+F)^{2} \sigma
$$

A comparison of (7), (6) and (2) yields the result that (7) is of the approximate magnitude

$$
w_{2}=\tau \partial w / \partial t
$$

We conclude that for processes which take a time $t$ large as compared to the relaxation time $\tau$ to effect an appreciable change in $w$ (or $\sigma$ ) the $S$-equation is a good approximation.

3. The diffusion model of chemical reactions. The diffusion current (6)

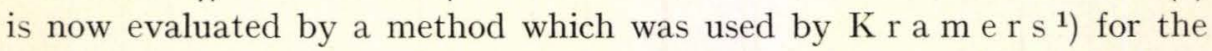
stationary state, but which may easily generalised. Expression (6) is written as

$$
w=-(k T / f) \exp (-U / k T) \cdot \partial\{\sigma \exp U / k T\} / \partial q
$$

Integration of (9) between points 1 and 2 (cf. fig. 1) yields

$$
\int_{1}^{2} w \exp U / k T \mathrm{~d} q=-(k T / f)\left\{\sigma_{2} \exp U_{2} / k T-\sigma_{1} \exp U_{1} / k T\right\}
$$

The discussion of the integral in (10) is facilitated by observing that for a model representing a chemical reaction the potential barrier is high as compared to $k T$. As a consequence the region near the top of the barrier gives the major contribution to the integral. On the other hand $w$ is very nearly constant in this region, as may be concluded by remarking that the particle density near the top of the barrier will not change very much during the course of time.

Equation (10) may be written as

$$
w \int_{\text {barrier }} \exp U / k T \mathrm{~d} q=-(k T / f)\left\{\sigma_{2} \exp U_{2} / k T-\sigma_{1} \exp U_{1} / k T\right\}
$$

The number of particles in state 1 may be defined as

$$
n_{1}=\int_{-\infty}^{t} \sigma \mathrm{d} q
$$

Now the majority of the particles in state 1 will be found near the bottom of the potential hole 1 . In that region the particles will be approximately 
distributed according to a Boltzmann distribution. Therefore $n_{1}$ may be written as

and $n_{2}$ as

$$
n_{1}=\sigma_{1} \exp U_{1} / k T \cdot \int_{\text {hole } 1} \exp (-U / k T) \mathrm{d} q
$$

$$
n_{2}=\sigma_{2} \exp U_{2} / k T \cdot \int_{\text {hole } 2} \exp (-U / k T) \mathrm{d} q
$$

Substitution of (14) and (13) in (11) and observing that $w=-\dot{n}_{1}$ yields $-\dot{n}_{1}=\left(k T / f \int_{\text {barrier }} \exp U / k T \mathrm{~d} q\right)$.

$$
.\left\{n_{1} / \int_{\text {hole } 1} \exp (-U / k T) \mathrm{d} q-n_{2} / \int_{\text {hole } 2} \exp (-U / k T) \mathrm{d} q\right\}
$$

This is an equation describing the transfer of particles between hole 1 and hole 2. It is of the form

$$
-\dot{n}_{1}=K_{1} n_{1}-K_{2} n_{2}
$$

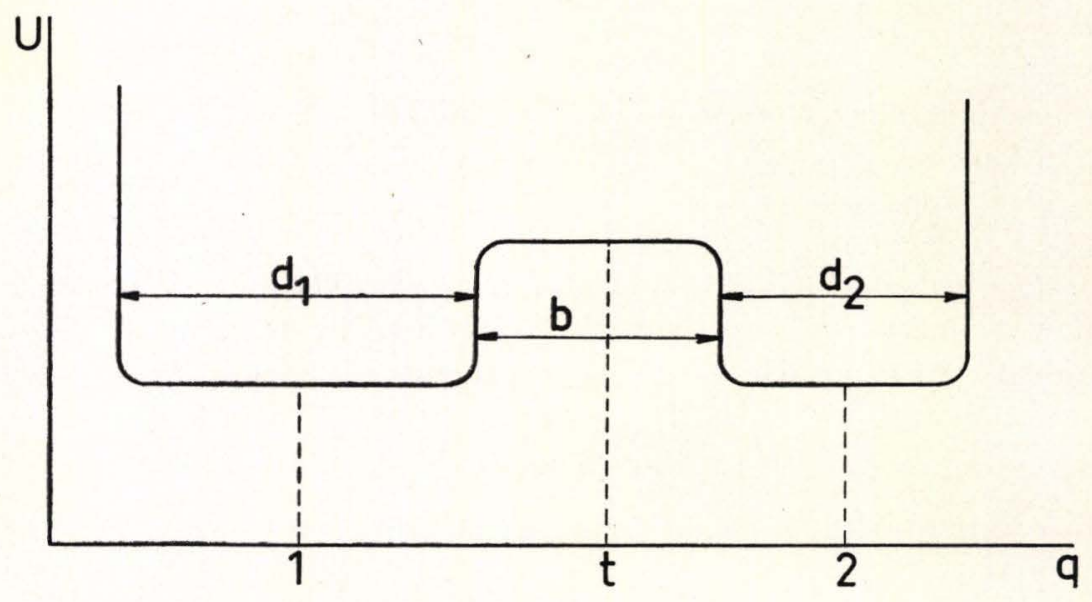

Fig. 2.

The reaction rates $K_{1}$ and $K_{2}$ depend on the form of the potential curve in the holes and near the top of the barrier

$$
\begin{aligned}
& K_{1}=k T /\left(f \int_{\text {barrier }} \exp U / k T \mathrm{~d} q \cdot \int_{\text {hole } 1} \exp (-U / k T) \mathrm{d} q\right) \\
& K_{2}=k T /\left(f \int_{\text {barrier }} \exp U / k T \mathrm{~d} q \cdot \int_{\text {hole } 2} \exp (-U / k T) \mathrm{d} q\right)
\end{aligned}
$$

As special cases we mention three examples:

1. Broad holes and barrier (fig. 2).

The reaction rates follow from (17) and (18)

$$
\begin{aligned}
& K_{1}=\left(k T / f b d_{1}\right) \exp \left(-U_{t}+U_{1}\right) / k T \\
& K_{2}=\left(k T / f b d_{2}\right) \exp \left(-U_{t}+U_{2}\right) / k T
\end{aligned}
$$

2. Edge shaped holes and barrier (fig. 3). 
Designating the forces at the slopes of the barrier by $F_{1}$ and $F_{2}$ the reaction rates become

$$
\begin{aligned}
& K_{1}=\left\{F_{1}^{2} F_{2} / k T f\left(F_{1}+F_{2}\right)\right\} \exp \left(-U_{t}+U_{1}\right) / k T \\
& K_{2}=\left\{F_{2}^{2} F_{1} / k T f\left(F_{1}+F_{2}\right)\right\} \exp \left(-U_{t}+U_{2}\right) / k T
\end{aligned}
$$

3. Parabolic form of holes and barrier.

Near 1:

Near 2:

Near $t$ :

$$
\begin{aligned}
& U=U_{1}+\left(\alpha^{2} / 2\right)\left(q-q_{1}\right)^{2} \\
& U=U_{2}+\left(\beta^{2} / 2\right)\left(q-q_{2}\right)^{2} \\
& U=U_{t}-\left(\gamma^{2} / 2\right)\left(q-q_{t}\right)^{2}
\end{aligned}
$$

The reaction rates are

$$
\begin{aligned}
& K_{1}=(\alpha \gamma / 2 \pi f) \exp \left(-U_{t}+U_{1}\right) / k T \\
& K_{2}=(\beta \gamma / 2 \pi f) \exp \left(-U_{t}+U_{2}\right) / k T
\end{aligned}
$$

The following conclusions may be drawn:

a. In all cases the reaction rates are proportional to the usual exponential function of the activation energy over $k T$. This is also true for reactions proceeding simultaneously in both directions.

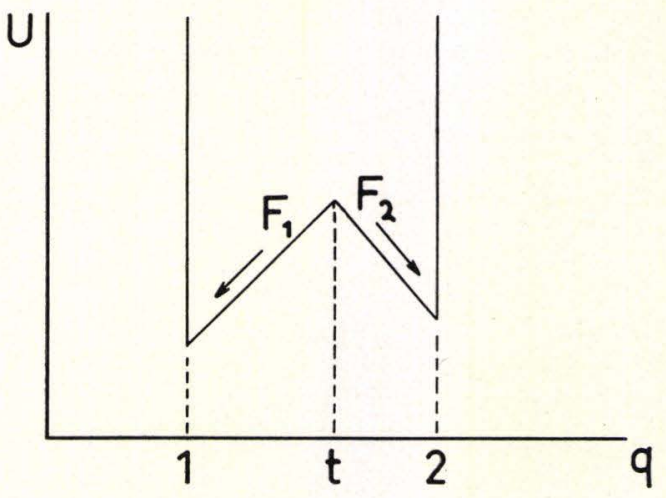

Fig. 3.

b. The temperature dependence of the non-exponential factor depends on the form of the potential curve. It may vary between proportionality with $T$ and $T^{-1}$.

c. The method which is based on a diffusion process is radically different from the transition state method which is based on a discussion of the state of equilibrium. It may be of interest to compare our results to those obtained by the transition state method (TSM). The TSM assumes temperature equilibrium and calculates the number of particles $\left(w_{T S M}\right)$ proceeding in one direction over the barrier. One finds

$$
w_{T S M}=\sigma_{t}(2 \pi m k T)^{-1 / 2} \int_{0}^{\infty}(p / m) \exp \left(-p^{2} / 2 m k T\right) \mathrm{d} p=(k T / 2 \pi m)^{1 / 2} \sigma_{t}
$$

where $\sigma_{t}$ is the particle density in the transition state. 
Introduction of (18) ...(13) yields the relation between $K_{T S M}$ and $K_{D T}$

$$
K_{D T} / K_{T S M}=\tau(2 \pi k T / m)^{1 / 2}\left\{\int_{\text {barrier }} \exp \left(U-U_{t}\right) / k T \mathrm{~d} q\right\}^{-1}
$$

where $D T$ means diffusion theory.

This relation is valid for both $K_{1}$ and $K_{2}$. The factor $\tau(2 \pi k T / m)^{1 / 2}$ represents the distance traveled by a particle starting with the gas kinetic velocity, while the integral in the denominator gives an estimate of the broadness of the barrier. Now the $S$-equation is a good approximation if the distance traveled by a particle before its systematic velocity is reduced to zero is small as compared to the region in which the diffusion takes place. We conclude that for processes for which the diffusion theory is valid, the resulting reaction rates are smaller than those following from the transition state method.

4. More dimensional models. A generalisation of the one-dimensional model of $\S 2$ is obtained by discussing a system of many (say $n$ ) degrees of freedom. The potential curve of fig. 1 is generalised in the following way. Holes 1 and 2 are many dimensional holes connected by a narrow pass. In and near the transition state the potential surface shows a negative curvature along a single coordinate (in the direction connecting the holes) and a positive curvature along all other coordinates.

The generalisation of expression (9) for the S-current is

$$
\mathbf{w}=-(k T / f) \exp (-U / k T) \cdot \operatorname{grad}\{\sigma \exp U / k T\}
$$

Integration along a curve between two points 1 and 2 yields

$$
\int_{1}^{2} \mathbf{w} \exp U / k T \mathbf{d s}=-(k T / f)\left\{\sigma_{2} \exp U_{\mathbf{2}} / k T-\sigma_{1} \exp U_{1} / k T\right\}
$$

Near the transition state the path of integration is chosen in such a way that $\mathbf{w}$ and $\mathbf{d s}$ are parallel. Then the left hand member of (25) may be written as (cf. (11))

$$
\int_{1}^{2} \mathbf{w} \exp U / k T \mathbf{d s}=w \int_{\text {barrier }} \exp U / k T \mathrm{~d} s
$$

where $\mathrm{d} s$ is a line-element near the top of the pass and parallel to $w$.

It should be realised that $w$ as obtained from (26) and (25) depends on the choice of the path of integration. The total number of particles passing from hole 1 to 2 is obtained by summing over all paths of integration, i.e. by the integration of $w$ over a more dimensional plane through the transition state and orthogonal to ds.

$$
-\dot{n}_{\mathbf{1}}=\int_{\text {all coordinates } \perp \mathbf{d s}} w \mathrm{~d} f
$$

The following result is obtained

$$
\begin{aligned}
& -\dot{n}_{1}=(k T / f) \int_{\text {all coordinates } \perp \mathrm{ds}} \mathrm{d} f /\left(\int_{\text {barrier }} \exp U / k T \mathrm{~d} s\right) . \\
& \quad .\left\{n_{1} / \int_{\text {hole } 1} \exp (-U / k T) \mathrm{d} V-n_{2} / \int_{\text {hole } 2} \exp (-U / k T) \mathrm{d} V\right\}
\end{aligned}
$$


Equation (27) is most easily discussed at the case of parabolic potentials in the neighbourhood of hole 1 (coordinates $q_{11}, q_{12}, \ldots, q_{1 n}$ ), hole 2 (coordinates $q_{21}, q_{22}, \ldots, q_{2 n}$ ) and the transition state (coordinates $q_{t 1}, q_{t 2}$, $\left.\ldots, q_{t n}\right)$

Near 1: $\quad U=U_{1}+\Sigma_{1}^{n}\left(\alpha_{i}^{2} / 2\right)\left(q_{i}-q_{1 i}\right)^{2}$

Near 2: $\quad U=U_{2}+\Sigma_{1}^{n}\left(\beta_{i}^{2} / 2\right)\left(q_{i}-q_{2 i}\right)^{2}$

Near $t: \quad U=U_{t}-\left(\gamma_{1}^{2} / 2\right)\left(q_{1}-q_{t 1}\right)^{2}+\Sigma_{2}^{n}\left(\gamma_{i}^{2} / 2\right)\left(q_{i}-q_{t i}\right)^{2}$

It is assumed that in the neighbourhood of the transition state $t$ the $S$-current is parallel to the coordinate $q_{1}$. Then the following reaction rates are obtained from (27)

$$
\begin{aligned}
& K_{1}=\left(\gamma_{1} \alpha_{1} \ldots \alpha_{n} / 2 \pi t \gamma_{2} \ldots \gamma_{n}\right) \exp \left(-U_{t}+U_{1}\right) / k T \\
& K_{2}=\left(\gamma_{1} \beta_{1} \ldots \beta_{n} / 2 \pi t \gamma_{2} \ldots \gamma_{n}\right) \exp \left(-U_{t}+U_{2}\right) / k T
\end{aligned}
$$

These expressions are analogous to those obtained from the one dimensional model (21). The analogy is best seen by the introduction of two apparent friction factors

$$
\begin{aligned}
& t_{1}=\hbar \gamma_{2} \ldots \gamma_{n} / \alpha_{2} \ldots \alpha_{n} \\
& t_{2}=t \gamma_{2} \ldots \gamma_{n} / \beta_{2} \ldots \beta_{n}
\end{aligned}
$$

If the pass is narrow as compared to holes 1 and $2\left(\gamma_{2} \ldots \gamma_{n} \gg \alpha_{2} \ldots \alpha_{n}\right.$ and $\beta_{2} \ldots \beta_{n}$ ), then $f_{1}$ and $f_{2}$ are much larger than $f$.

We conclude that for a narrow pass the reaction rates are much smaller than those obtained from the one-dimensional model. This is not the case if the curvatures in the pass and the holes are equal.

Received 14-12-55.

\section{REFERENCES}

1) Kramers, H. A., Physica 7 (1940) 284.

2) Brinkm a n, H. C., Physica 22 (1956) 29. 